\title{
The Utility of Pyrogenic Cytokine (IL-1 $\beta$, TNF- $\alpha$ and IL-6) Detection In Risk Stratification of Critically lll Febrile Patients in Emergency Department
}

Francesco Travaglino ${ }^{1,3}$, Gerardo Salerno ${ }^{2,3}$, Veronica Russo ${ }^{1,3}$, Mariateresa Corsetti ${ }^{2,3}$, Rosaria D'Urso ${ }^{2,3}$, Patrizia Cardelli ${ }^{2,3}$, Maria Rosaria Torrisi ${ }^{2,3}$, Vincenzo Visco ${ }^{2,3^{*}}$ and Salvatore Di Somma ${ }^{1,3}$

${ }^{1}$ Emergency Department, Faculty of Medicine and Psychology University of Rome "Sapienza", Rome, Italy

${ }^{2}$ Department of Clinical and Molecular Medicine, Istituto Pasteur-Fondazione Cenci Bolognetti, Faculty of Medicine and Psychology University of Rome "Sapienza", Rome, Italy

${ }^{3}$ Sant'Andrea Hospital, Rome, Italy

*Corresponding author: Vincenzo Visco, Sant'Andrea Hospital, Via di Grottarossa 1035, 00189 Rome, Italy, Tel: +39 0633776771; E-mail: vincenzo.visco1@uniroma1.it

Rec date: Jul 18, 2014; Acc date: Aug 28, 2014; Pub date: Sep 01, 2014

Copyright: (c) 2014 Travaglino F, et al. This is an open-access article distributed under the terms of the Creative Commons Attribution License, which permits unrestricted use, distribution, and reproduction in any medium, provided the original author and source are credited.

\begin{abstract}
Objective: Aim of our study was to evaluate the prognostic value of pyrogenic cytokines (IL-1 $\beta$, TNF- $\alpha$ and IL-6) detection associated with Mid regional pro-Amedullin (MR-proADM) and the APACHE II score in febrile patients admitted to the Emergency Department (ED).

Material: 64 patients in the Emergency Room (ER) during a period of 12 months with body temperature $>37^{\circ} \mathrm{C}$ were recruited for this study. In order to compare MR-proADM and cytokine values, a control group of 40 healthy volunteers was enrolled. For each subject, the APACHE II score was calculated.

Result: MR-proADM and cytokines were significantly higher in patients compared to controls $(p<0.0001)$. When APACHE II score was correlated to MR-proADM and cytokines and grouped into quartile, it showed a significant increase in TNF- $\alpha$ and IL-6 levels $(p<0.0001)$. A significant stepwise increase in MR-proADM in accordance with IL-6 quartile levels was observed $(p<0.0001)$. The receiver operating characteristic $(R O C)$ curve showed the ability of those combined biomarkers to predict hospitalization.
\end{abstract}

Conclusion: The combined use of MR-proADM and cytokines can help the febrile patient's management in the ED, predicting a subsequent hospitalization of such individuals.

Keywords: Fever; MR-proADM; Cytokines

\section{Introduction}

Fever is one of the most typical signs encountering patients in access to the Emergency Department (ED) and it is highly suggestive that a bacterial infection is involved [1]. It is an adaptive defense that is necessary for the protection and survival of the host in the presence of pathogenic agents. The signaling pathways governing the body temperature enhancement are complex and still remain unclear. Nevertheless symptoms and signs of different infective diseases are often overlapping and rarely specific [2]. For those reasons, an early identification of the fever etiology is quite complicated and should be extremely useful in the management of patients presenting to the ED [3]. To date, the combined use of clinical parameters and classical laboratory biomarkers, such as C-reactive protein (CRP) and leukocyte count, represents yet the current strategy to distinguish between various causes of fever [4]. In addition, Adrenomedullin (ADM), a 52 amino acids comprising peptide with several activities, the most relevant being its vasodilatative function, has been studied in febrile patients because it could be helpful in the evaluation of sepsis' diagnosis and prognosis [5,6]. In a recent study, our group demonstrated the utility of Mid-regional fragment proadrenomedullin (MR-proADM), which is the more stable part of
ADM included between the aminoacids 49-52, in the risk stratification of critically ill patients presenting for fever in the ED [2]. In particular, MR-proADM was identified as a biomarker of prognostic value, helpful mainly in individual risk assessment of septic patients [7].

However, it is known that the fever is also mediated by the release of several pyrogenic molecules, which are crucial for the systemic host response to infection. Microorganism invasion stimulates a large panel of cells -including monocytes/macrophages and neutrophils- to synthesize mediators, called proinflammatory cytokines [8]. Feverinducing exogenous pyrogens, such as lipopolysaccharide, cause the production of soluble proteins that act as endogenous pyrogens on the central nervous system [9]. Those molecules play a central role in inducing an increase of the temperature set point through a cascade of events priming the production of key inflammatory mediators such as prostaglandins (PGs), especially of the PG class E2 (PGE2) [10-12]. Candidate endogenous pyrogens include the proinflammatory cytokines IL-1 $\beta$, TNF- $\alpha$ and IL- 6 , showing several properties. It is well documented that these molecules enhance the body temperature, trigger endothelial cell activities, contribute to the recruitment of circulating polymorphonuclear cells (PMNs) and enter the circulatory system [13].

IL-1 is the primary and the most important cytokine showing pyrogenic properties [14] and particularly IL- $1 \beta$ seems to be the major 
Citation: Travaglino F, Salerno G, Russo V, Corsetti M, Urso RD, et al. (2014) The Utility of Pyrogenic Cytokine (IL-1 $1 \beta$, TNF- $\alpha$ and IL-6) Detection In Risk Stratification of Critically III Febrile Patients in Emergency Department. J Mol Biomark Diagn 5: 188. doi: $10.4172 / 2155-9929.1000188$

Page 2 of 5

endogenous pyrogen in viral infections [9]. TNF- $\alpha$ is a proinflammatory cytokine, able to stimulate the acute phase response, being highly pyrogenic in humans, that is mainly produced by macrophages and supports similar biologic functions of IL-1 $\beta$ [15]. Even though IL-6 is considered a potent proinflammatory cytokine, it requires much higher amounts than IL-1 and TNF to induce fever. It has been suggested that IL-6 is a downstream mediator of fever from those two cytokines, whose secondary synthesis triggers the mechanisms producing a temperature enhancement through the secretion of PGE2 [12].

Sepsis-induced organ failure is caused by activation of infective pathway initiated by micro-organisms and systemic sepsis releases some cytokines finally causing septic shock $[16,17]$. Numerous studies have evaluated the role of cytokine determinations as early diagnostic markers in the pathophysiology of sepsis $[13,18]$. Considering that febrile critically ill patients still represent an intriguing challenge for the ED, it is crucial to develop innovative strategies in order to facilitate rapid diagnosis, appropriate treatment and to better define their disposition.

The aim of this work was to analyze the prognostic burden of cytokine plasma levels in association with MR-proADM in a cohort of well-defined febrile patients, in comparison with the well known disease severity index score Acute Physiology And Chronic Health Evaluation (APACHE II) score. This prognostic score is obtained by the combination of parameters measured during the initial hours after the access to the ED and is used to predict mortality of critically ill patients [19].

\section{Materials and Methods}

The present work was conducted by a subanalysis of our previous published study [2], in the ED of the Sant'Andrea University Hospital in Rome, Italy. This study was approved by the Hospital Ethics Committee and all patients provided written informed consent. An observational study, with an enrollment period of 12 months (from October 2009 to October 2010), including 128 patients, 64 of them referred to the ED with fever (body temperature $>37^{\circ} \mathrm{C}$ ) and a suspicion of severe infection such as sepsis, lower respiratory tract infections, urinary tract infections and others, was performed. Patients younger than 18 years old were excluded.

After ED admission, each patient was clinically examined, a blood sample for biomarkers was obtained from peripheral blood catheter, immediately separated into plasma and stored at $-80^{\circ} \mathrm{C}$ for the following analysis.

MR-proADM and cytokines were measured in $50 \mu$ of plasma by a Time-Resolved Amplified Cryptate Emission (TRACE) technology assay -using kits designed for automated sandwich immunofluorescent assay of MR-proADM (KRYPTOR, Brahms, Germany) - and a multiplex biochip array in Evidence equipment (Randox Labs, Ltd Crumlin, UK), respectively.
For each patient, the APACHE II score was calculated and a standardized form was completed, as previously reported [2].

A control group of 40 healthy volunteers -who had no past medical history and were not on medication, older than 18 years- was considered for the comparison of MR-proADM and cytokines values between patients and the control group itself.

Concerning statistical analysis, continuous variables are expressed as mean \pm standard deviation (SD) or median with interquartile range (IQR) in parenthesis, unless stated otherwise. Two-group comparisons were calculated by the Mann-Whitney U test or Unpaired t-test. For multigroup comparisons, the Kruskal-Wallis test was used. Correlation analyses were performed using Spearman rank correlation. To determine the ability of the biomarkers to predict hospitalization, a receiver operating characteristic (ROC) curve was calculated. All statistical tests were two-tailed, and $\mathrm{p}<0.05$ was considered statistically significant.

\section{Results}

Patients' characteristics are shown in Table 1a. The values of MRproADM, IL-1 $\beta$, TNF- $\alpha$, IL-6 and APACHE II score are reported in Table $1 b$.

The correlation between the APACHE II score, MR-proADM and the different cytokines levels were evaluated and divided according to the final ED's diagnosis (data not shown). IL-1 $\beta$, TNF-aand IL-6 values were compared between healthy subjects (control group) and patients (Figure 1a). Cytokine levels were significantly increased in patients compared to healthy individuals $(p<0.0001)$. The APACHE II score, expressed in predicted mortality percentage, was grouped into quartiles and compared respectively with IL-1 $\beta$, TNF- $\alpha$ and IL- 6 levels. IL- 6 and TNF- $\alpha$ levels were significantly stepwise increased in accordance with the APACHE II quartiles $(p<0,0001)$, whereas IL- $1 \beta$ levels were not significantly modified. The dashed line represents the median in controls (Figure 1b). MR-proADM levels showed a correlation with IL-6 levels in the patients' group $(r=0.57, p<0,0001)$. In all patients there was a statistically significant stepwise increase in MR-proADM levels in accordance with IL-6 values; the dashed line represents the median in controls. $(p<0,0001$ - Figure $1 b)$. Possible explanations of the correlations showed above involve the timedepending cytokine production during the temperature enhancement.

Then we evaluated the ability of MR-proADM and the three cytokines to predict hospitalization in patients admitted to our emergency departments complaining of fever.

The area under the curve (AUC) of MR-proADM was 0.776 $(p<0.0001)$, AUC of IL- 6 was $0.74(p<0,0001)$, AUC of IL- $1 \beta$ was 0.71 $(p=0,0001)$, AUC of TNF- $\alpha$ was $0.71(p=0,0001)$ (Figure $2 a)$. The combined use of total biomarkers showed an AUC of $0.80(p<0,0001)$ (Figure 2a). Finally, we performed a matrix correlation of the different variables, whose values are expressed as colored circles of different sizes and shown in Figure 2b.

\begin{tabular}{|l|l|l|l|}
\hline a) & All Patients & Control & $P$ value \\
\hline Gender & $\begin{array}{l}35 \text { male } \\
29 \text { female }\end{array}$ & $\begin{array}{l}23 \text { male } \\
17 \text { female }\end{array}$ & \\
\hline Age-Years (MeanSD) & 5819 & 5515 & 0.72 \\
\hline
\end{tabular}


Citation: Travaglino F, Salerno G, Russo V, Corsetti M, Urso RD, et al. (2014) The Utility of Pyrogenic Cytokine (IL-1 1 , TNF- $\alpha$ and IL-6) Detection In Risk Stratification of Critically III Febrile Patients in Emergency Department. J Mol Biomark Diagn 5: 188. doi: $10.4172 / 2155-9929.1000188$

Page 3 of 5

\begin{tabular}{|l|l|l|l|}
\hline TemperatureC (MeanSD) & 38.60 .6 & 36.40 .3 & 0.03 \\
\hline b) & & $0.50(0.40-0.58)$ & $<0.0001$ \\
\hline $\begin{array}{l}\text { MR-proADM (nmol/mL) } \\
\text { (Median, IQR) }\end{array}$ & $0.82(0.54-1.62)$ & $0.50(0.40-0.60)$ & $<0.0001$ \\
\hline $\begin{array}{l}\text { IL-1 } \beta(\mathrm{pg} / \mathrm{ml}) \\
\text { (Median, IQR) }\end{array}$ & $6.6(2.40-12.50)$ & $0.74(0.68-0.78)$ & $<0.0001$ \\
\hline $\begin{array}{l}\text { TNF- } \alpha(\mathrm{pg} / \mathrm{ml}) \\
\text { (Median, IQR) }\end{array}$ & $117.3(36.20-188.90)$ & $0.42(0.23-0.60)$ & $<0.0001$ \\
\hline $\begin{array}{l}\text { IL-6 }(\mathrm{pg} / \mathrm{ml}) \\
\text { (Median, IQR) }\end{array}$ & $132.5(36.20-188.90)$ & & $<0.0001$ \\
\hline APACHE II (\%)(Median, IQR) & $12.10(6.02-21)$ & & \\
\hline
\end{tabular}

Table 1: Patient's characteristics

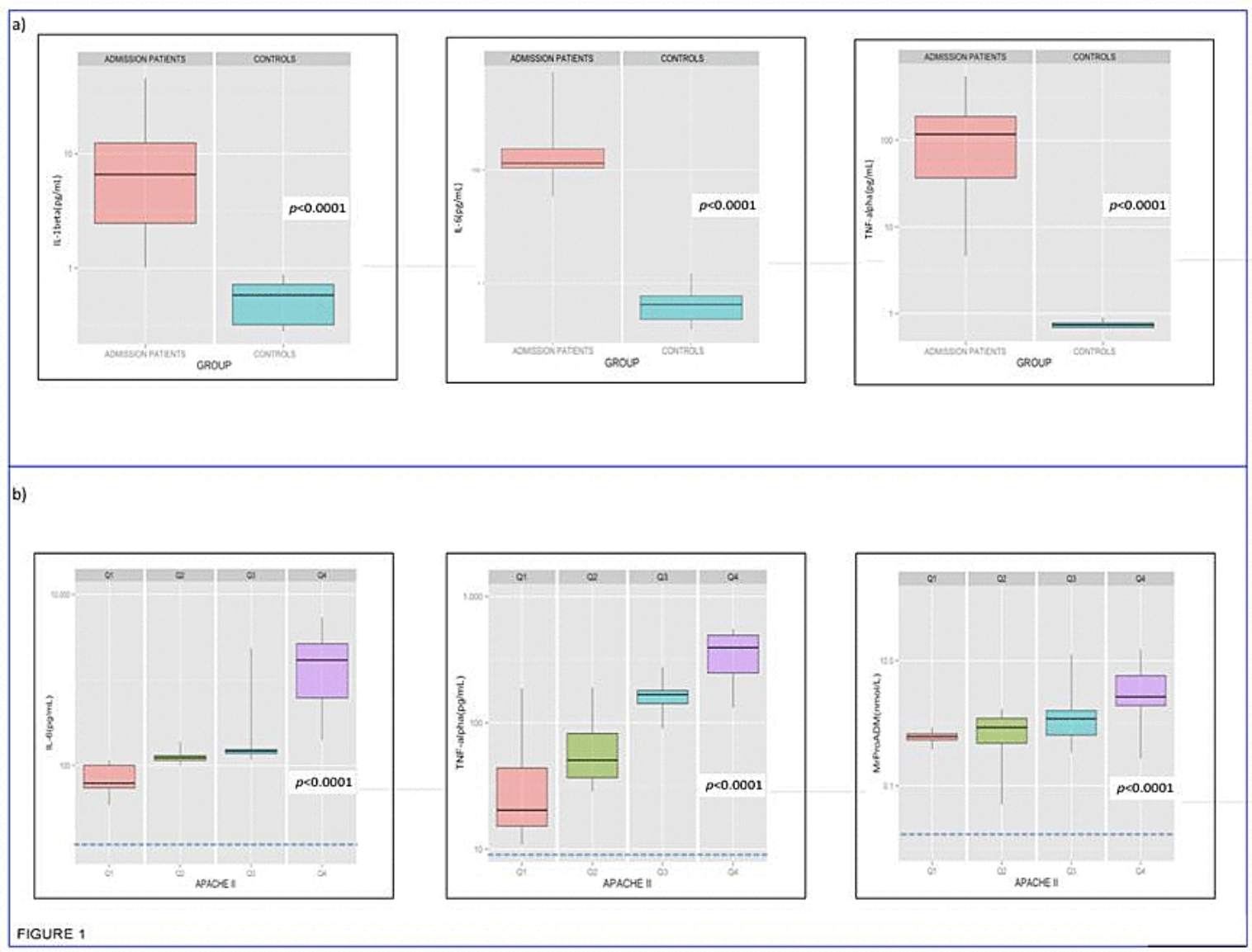

Figure 1:a) Levels of Cytokines at admission expressed as $\mathrm{pg} / \mathrm{ml}$. 1b) Correlation between IL-6 and APACHE II quartiles, TNF- $\alpha$ and APACHE II quartiles. Correlation between MR-proAMD (expressed as mmol/L) and IL-6 quartiles 
Citation: Travaglino F, Salerno G, Russo V, Corsetti M, Urso RD, et al. (2014) The Utility of Pyrogenic Cytokine (IL-1 1 , TNF- $\alpha$ and IL-6) Detection In Risk Stratification of Critically III Febrile Patients in Emergency Department. J Mol Biomark Diagn 5: 188. doi: 10.4172/2155-9929.1000188

Page 4 of 5

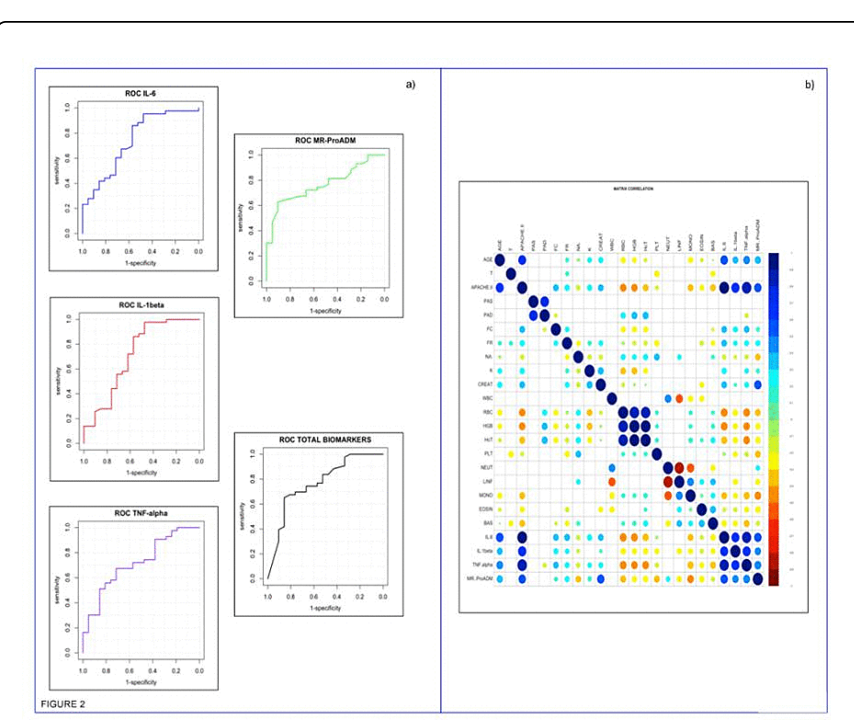

Figure 2: a) ROC curves for predicting patient's hospitalization.2b) Matrix Correlation. The bar with the range of colors (on the right) represents the different correlation levels (positive and negative)

\section{Discussion}

The difficulties routinely encountered in the management of patients affected by nonspecific fever and recruited in the ED are well known and frequently a matter of debate. For example, the Adrenomedullin precursor, named MR-proADM (45-92), is present in large amounts of septic shock patients and, also for this reason, was suggested as a novel infection biomarker to facilitate the management of patients who are admitted in the ED with fever $[2,6]$.

Nevertheless taking care of the febrile patients is always lacking of reliable biomarkers, because it will be presumably very difficult to discover one single molecule capable to satisfy all the present needs for an early and accurate diagnostic approach of those patients. In the light of these considerations, it could be expected that only the combination of several biomarkers, supported by convincing and appropriate statistical methods, may represent an operational goal in this field. Therefore, increasing evidences suggest that the inflammatory profile of patients with fever may influence the course of different pathologies and that such human immunological status is reflected by the release of a consistent number of molecules in the plasma, including pro-inflammatory and pyrogenic cytokines, as IL-1 $\beta$, TNF- $\alpha$ and IL-6 [20].

Hence, the aim of the present study was to evaluate the prognostic value of the major pyrogenic cytokines (i.e. IL- $1 \beta$, TNF- $\alpha$ and IL- 6 ) in febrile patients in the ED in association with MR-proADM and in comparison with the APACHE II severity index score, in order to predict their subsequent hospitalization.

As expected, both the MR-proADM and cytokine plasma levels were significantly higher compared to healthy donors [2,21]. Interestingly, IL- 6 and TNF- $\alpha$ but not IL- $1 \beta$ levels were significantly stepwise increased in accordance with the APACHE II quartiles, whereas MR-proADM levels showed a strong correlation only with IL-6. Thinking that IL-6 is a downstream mediator of fever and its secretion was described to be under the control of IL-1 and TNF [22], we suggest that a possible explanation is probably dependent on the different steps of the fever-inducing mediators release. In fact, IL- $1 \beta$ and TNF- $\alpha$ may be involved in the initial host response, whereas IL- 6 is only secondarily secreted [12] and such variations could justify the different correlations with the APACHE II quartiles and MR-proADM levels. At the light of these considerations, it could be presumed that our results are linked to the exact beginning of the febrile attack, because the in vivo changes of proinflammatory cytokines production is time-dependent.

However, our data support the prognostic role of MR-proADM and cytokine detection and that their combined use can predict a subsequent hospitalization of febrile patients accessing the ED. The rational use of these molecules could be expected to result in several advantages, such as faster diagnosis and prognosis, more accurate risk stratification, and optimization of treatment, with consequent benefit to the patient. A broader multi-hospital study will permit to further elucidate the exact mechanisms able to induce MR-proADM and pyrogenic cytokines production in febrile patients.

\section{References}

1. Limper M, EeftinckSchattenkerk D, de Kruif MD, van Wissen M, Brandjes DP, et al. (2011) One-year epidemiology of fever at the Emergency Department. Neth J Med 69: 124-128.

2. Travaglino F, De Berardinis B, Magrini L, Bongiovanni C, Candelli M, et al. (2012) Utility of Procalcitonin (PCT) and Mid regional proAdrenomedullin (MR-proADM) in risk stratification of critically ill febrile patients in Emergency Department (ED). A comparison with APACHE II score. BMC Infect Dis 12: 184.

3. Schuetz P, Christ-Crain M, Thomann R, Falconnier C, Wolbers M, et al. (2009) Effect of procalcitonin-based guidelines vs standard guidelines on antibiotic use in lower respiratory tract infections: the ProHOSP randomized controlled trial. JAMA 9: 1059-1066.

4. Bossink AW, Groeneveld AB, Thijs LG (1999) Prediction of microbial infection and mortality in medical patients with fever: plasma procalcitonin, neutrophilic elastase-alpha1-antitrypsin, and lactoferrin compared with clinical variables. Clin Infect Dis 29: 398-407.

5. Hinson JP, Kapas S, Smith DM (2000) Adrenomedullin, a multifunctional regulatory peptide. Endocr Rev 21: 138-167.

6. Struck J, Tao C, Morgenthaler NG, Bergmann A (2004) Identification of an Adrenomedullin precursor fragment in plasma of sepsis patients. Peptides 25: 1369-1372.

7. Christ-Crain M, Morgenthaler NG, Struck J, Harbarth S, Bergmann A, et al. (2005) Mid-regional pro-adrenomedullin as a prognostic marker in sepsis: an observational study. Crit Care 9: R816-824.

8. Dinarello CA (1999) Cytokines as endogenous pyrogens. J Infect Dis 179 Suppl 2: S294-304.

9. Alcamí A, Smith GL (1996) A mechanism for the inhibition of fever by a virus. ProcNatlAcadSci U S A 93: 11029-11034.

10. Saper CB, Breder CD (1994) The neurologic basis of fever. N Engl J Med 330: 1880-1886.

11. Dinarello CA, Gatti S, Bartfai T (1999) Fever: links with an ancient receptor. CurrBiol 9: R147-150.

12. Netea MG, Kullberg BJ, Van der Meer JW (2000) Circulating cytokines as mediators of fever. Clin Infect Dis 31 Suppl 5: S178-184.

13. Cho SY, Choi JH (2014) Biomarkers of sepsis. Infect Chemother 46: 1-12.

14. Tewari A, Buhles WC Jr, Starnes HF Jr (1990) Preliminary report: effects of interleukin-1 on platelet counts. Lancet 336: 712-714.

15. Dinarello CA (1991) Inflammatory cytokines: interleukin-1 and tumor necrosis factor as effector molecules in autoimmune diseases. CurrOpinImmunol 3: 941-948. 
Citation: Travaglino F, Salerno G, Russo V, Corsetti M, Urso RD, et al. (2014) The Utility of Pyrogenic Cytokine (IL-1 $\beta$, TNF- $\alpha$ and IL-6) Detection In Risk Stratification of Critically III Febrile Patients in Emergency Department. J Mol Biomark Diagn 5: 188. doi: $10.4172 / 2155-9929.1000188$

Page 5 of 5

16. Kothari N, Bogra J, Abbas H, Kohli M, Malik A, et al. (2013) Tumor necrosis factor gene polymorphism results in high TNF level in sepsis and septic shock. Cytokine 61: 676-681.

17. Hamzic N, Tang Y, Eskilsson A, Kugelberg U, Ruud J, et al. (2013) Interleukin- 6 primarily produced by non-hematopoietic cells mediates the lipopolysaccharide-induced febrile response. Brain BehavImmun 33: 123-130.

18. de Bont ES, Martens A, van Raan J, Samson G, Fetter WP, et al. (1993) Tumor necrosis factor-alpha, interleukin-1 beta, and interleukin-6 plasma levels in neonatal sepsis. Pediatr Res 33: 380-383.

19. Knaus WA, Draper EA, Wagner DP, Zimmerman JE (1985) APACHE II: a severity of disease classification system. Crit Care Med 13: 818-829.
20. Reinhart K, Bauer M, Riedemann NC, Hartog CS (2012) New approaches to sepsis: molecular diagnostics and biomarkers. ClinMicrobiol Rev 25: 609-634.

21. Van Damme J, Opdenakker G, Simpson RJ, Rubira MR, Cayphas S, et al. (1987) Identification of the human $26-\mathrm{kD}$ protein, interferon beta 2 (IFN-beta 2), as a B cell hybridoma/plasmacytoma growth factor induced by interleukin 1 and tumor necrosis factor. J Exp Med 165: 914-919.

22. Zetterström M, Sundgren-Andersson AK, Ostlund P, Bartfai T (1998) Delineation of the proinflammatory cytokine cascade in fever induction. Ann N Y AcadSci 856: 48-52. 